\title{
Modelos de gobernanza y desarrollo sustentable: Una propuesta instrumental para los municipios de Puebla
}

\author{
Models of governance and sustainable development: An instrumental \\ proposal for the municipalities of Puebla
}

\author{
José Antonio MEYER RODRÍGUEZ \\ Benemérita Universidad Autónoma de Puebla \\ joseantonio.meyer@gmail.com \\ Margarita LUMBRERAS HERNÁNDEZ \\ Benemérita Universidad Autónoma de Puebla \\ margarita.lumbreras@correo.buap.mx
}

Recibido: 6 de marzo de 2018 Aceptado: 21 de abril 2018

\section{Resumen}

A partir de los principios de un gobierno incluyente, el presente trabajo desarrolla un modelo de gobernanza local para los municipios del Estado de Puebla. En un contexto de graves contrastes, donde las principales evaluaciones sobre desarrollo humano destacan rezagos en materia salud, educación, nutrición y vivienda, el modelo plantea nuevas formas de gestión pública municipal con la participación de la ciudadanía y un proceso constante de evaluación y rendición de cuentas. El modelo tiene un enfoque sistémico, aglutinador, abierto y democrático que busca alcanzar el pleno ejercicio de la gobernanza y producir mejoras sustantivas en las condiciones de vida de las diversas comunidades del territorio.

\section{Abstract}

Based on the principles of an inclusive government, this work develops a local governance model for the municipalities of Puebla state. In a context of serious contrasts, where the main evaluations on human development highlight lags in health, education, nutrition and housing, the model proposes new forms of municipal public management with the participation of citizens and a constant evaluation process and accounts rendering. The model has a 
systemic, unifying, open and democratic approach that seeks to achieve the full exercise of governance and produce substantive improvement in the living conditions of the various communities of different territories.

Palabras clave: Gobierno incluyente; gobernanza participativa y gestión pública Key Words: Inclusive Government; Participative Governance and Public Management

\section{Introducción}

El Estado de Puebla se ha constituido en una de las entidades de México con mayor nivel de contrastes en términos económicos, políticos y sociales. Ello se debe, en primer término, a que en el $1.75 \%$ del territorio nacional (34 mil 251 kilómetros cuadrados de superficie) se asienta actualmente un conglomerado demográfico con uno de los índices de crecimiento poblacional más altos a nivel nacional (6 millones 169 mil habitantes) (INEGI, 2015). En segundo, que pese a contar con un índice de crecimiento económico superior a la media nacional los beneficios se concentran mayormente en tres zonas metropolitanas compuestas por 25 municipios, mientras que en el resto del territorio (197 municipios) existe una densidad poblacional baja, con una actividad agrícola básica y de extracción decreciente, serios rezagos en materia de educación y salud y relaciones sociales supeditadas a un fuerte control político de los partidos políticos y los cacicazgos tradicionales (Székely, Rodríguez, Flores, Leyson y Mendoza, 2017).

La metropolización y la urbanización desordenadas de los últimos años, que han tendido a determinar mayormente las diferencias territoriales desplazando a diversos municipios con vocación agrícola, se concentra en el $12 \%$ de los municipios de la entidad. Ello ha significado la expansión creciente de las zonas urbanas y una mayor concentración demográfica por actividades económicas más remunerativas, así como un acceso diversificado a los beneficios de carácter social. La tendencia se ha incrementado en las últimas tres décadas por una serie de intervenciones gubernamentales que han incidido directamente en la inversión pública, la infraestructura y las dinámicas socio-económicas en regiones 
determinadas de la entidad (CONEVAL, 2017). De esta manera, si bien a nivel del estado se destacan crecientes Índices de Desarrollo Industrial (SE, 2017) que ubican a Puebla en la 9ª posición nacional (con un 3.2\% de participación en el Producto Interno Bruto) y el 11ㅇlugar en el país por su participación del 2.9\% del Valor Agregado Censal Bruto, también se manifiestan bajos Índices de Desarrollo Humano (PNUD, 2017) en diversas regiones del territorio que la ubican en el lugar No. 29 a nivel nacional con algunos municipios por debajo de la línea básica de pobreza y otros más con pobreza extrema y marginación. Por tanto, aunque Puebla reporta un nivel de desarrollo humano medio, comparable con países como Colombia y Turquía, también manifiesta municipios con condiciones semejantes a las de Ruanda.

El tamaño de la economía del estado se debe, en gran parte, al tamaño de la población con un 5.14\% del total nacional. Sin embargo, la concentración de las actividades productivas en ciertas zonas hace que la economía no sea tan grande cuando se le mide en términos per cápita. Esa posición baja al lugar No. 27 cuando se estima su participación en el Producto Interno Bruto per cápita en estados sin actividad petrolera con respecto al valor nacional $(2.1 \%)$ y si se le compara con la precariedad en los indicadores de bienestar social en territorios determinados (Índice de Progreso Social, 2017). En ese sentido, de acuerdo con cifras del Programa de las Naciones Unidas para el Desarrollo (PNUD, 2017), entre 2008 y 2010 se registró un IDH entre el 0.6897 y el 0.7106 ubicando a Puebla en el lugar No. 27 a nivel nacional. Los municipios con mejor puntuación fueron los de carácter urbano como Juan Galindo (0.730), Teziutlán (0.742), San Andrés Cholula (0.745), San Martín Texmelucan (0.749), Zaragoza (0.750), Teteles de Ávila (0.768), San Pedro Cholula (0.775), Cuautlancingo (0.782) y Puebla (0.830). Mientras que los de menor puntuación y mayor pobreza fueron los de tipo rural como Eloxochitlán (0.437), Chichiquila (0.470), Quimixtlán (0.471), Vicente Guerrero (0.472), Chilchotla (0.475), Coyomeapan (0.478), Zoquitlán (0.483), Tepetzintla (0.486), Chiconcuautla (0.490), y San Felipe Tepatlán (0.490). 
Las relaciones entre las dimensiones del Índices de Desarrollo Humano (IDH) y algunas metas de los Objetivos del Desarrollo del Milenio (ONU, 2015) enfatizan también la existencia de variadas realidades en una misma entidad y múltiples disparidades que pueden identificarse al dividir el territorio en regiones o concentrado de municipios. La región de Angelópolis presenta los niveles de IDH más alto con un $26.7 \%$ mayor a la región del Valle Serdán. De igual forma, el IDH del municipio de Puebla es $86.7 \%$ superior al de Teopantlán. Esta tendencia se repite al examinar los indicadores de los ODS. Así, por ejemplo, si se toma el indicador de la matrícula escolar a nivel estatal se identifica que ya se ha cumplido la meta con anticipación. No obstante, 191 municipios no alcanzarán esa meta ni siquiera en 2020 y hay algunos otros que si pudieran alcanzarla si duplican sus esfuerzos y mantienen la pauta de cumplimiento en por lo menos 5 años continuos. De igual forma, aunque 45 municipios subieron su meta al reducir en $1 / 3$ la tasa de mortalidad infantil en 2015, enfrentan también retos importantes como la erradicación de la pobreza extrema y el hambre, la enseñanza primaria universal, la igualdad de género y el empoderamiento de la mujer, la mortalidad de los niños menores de 5 años, la salud materna, el combate al VIH/Sida, el paludismo y otras enfermedades, la sostenibilidad del medio ambiente y las alianzas para incentivar su desarrollo.

Como ha sucedido con otras regiones del país, el desarrollo urbano se ha generado luego de la segunda mitad del siglo XX como resultado del crecimiento económico y la industrialización, donde las mejores condiciones de empleo, educación, salud, vivienda y nutrición generalmente se ubican en los municipios urbanos. En Puebla hubo un predominio de la población rural entre las décadas de 1900 y 1960, pero en las cuatro últimas décadas del siglo XX se ha manifestado un incremento sustantivo de la población asentada en zonas urbanas como resultado de la industrialización. Las cifras muestran que mientras la población urbana se incrementó en un $75 \%$, la rural descendió en un $25 \%$. Ese gran 
crecimiento de la población se concentró, como lo ha referido Ornelas (2012:22), "en regiones específicas del territorio, muy destacadamente en la zona del Valle de Puebla donde se ha constituido una amplia zona metropolitana que incluye municipios de los estados de Puebla y Tlaxcala".

El Consejo Nacional de Población (2016) ha modificado a través del tiempo la delimitación de las zonas metropolitanas del Estado de Puebla en función de la dinámica seguida por los conglomerados poblacionales. De esta manera, reconoce que actualmente existen dos zonas metropolitanas en el interior del estado y una más que se comparte con el vecino estado de Tlaxcala. La más importante es precisamente la Zona Metropolitana PueblaTlaxcala (ZMPT) que cuenta con 2 millones 728 mil 790 habitantes distribuidos en una extensión de 2 mil 392 km2 y presenta una densidad media urbana de 76.6 habitantes por hectárea. Este conglomerado urbano se compone por 38 municipios (18 de Puebla y 20 de Tlaxcala), entre los que se encuentran Puebla (1 millón 539 mil 819 habitantes), San Martín Texmelucan (141 mil 112 habitantes), San Pedro Cholula (120 mil 459 habitantes), Amozoc (100 mil 964 habitantes), San Andrés Cholula (100 mil 439 habitantes), Chiautzingo (79 mil 153 habitantes), Tepeyanco (69 mil 615 habitantes) y Huejotzingo (63 mil 457 habitantes), todos ellos localizados en Puebla excepto el penúltimo. Cabe señalar que la situación económica de esta zona es mejor que la de todo el Estado de Puebla, con un valor de 0.73 en el Atlas de Complejidad Económica (2016) y el lugar No. 18 en el ranking de las entidades del país. Sin embargo, el peso económico de esta zona no logra aún incidir en forma significativa en la generación de una actividad económica al interior de la entidad y la actividad económica de las zonas del interior tampoco contribuye a mejorar el potencial productivo de la entidad (García, 2017).

La zona metropolitana Puebla-Tlaxcala es considerada como la cuarta área territorial urbana del país en términos poblacionales, después de las del Valle de México, Guadalajara y 
Monterrey. Aunque los criterios empleados por el INEGI y la CONAPO para delimitar las zonas metropolitanas en México consideran las relaciones económicas y sociales entre los municipios conurbados, la interacción intermunicipal en términos de movilidad y la dinámica entre los municipios y la ciudad central, en el caso de la zona metropolitana Puebla-Tlaxcala la ciudad referente es Puebla capital, en la cual se asienta más de la mitad de los habitantes de la metrópoli. Esta zona creció a una tasa promedio anual del 6\% entre 2009 y 2014 , donde Puebla concentró el 95\% de la actividad económica y el 97\% de la inversión. En los municipios de Tlaxcala el $25 \%$ de la actividad es la fabricación de productos a base de minerales y construcción, así como la industria alimentaria. El $44 \%$ del PIB de la parte poblana proviene de la fabricación de equipo de transporte y la manufactura, construcción, comercio y servicios empresariales que concentran la mayoría de los empleos registrados. En 2016 Puebla fue la cuarta ciudad del país en población (casi 3 millones de habitantes), por tamaño de actividad económica (436.6 miles de millones de pesos) y tamaño de población económicamente activa (1 millón37 mil trabajadores), así como la tercera en tamaño de extensión urbana (621 km2). Sin embargo, fue también la número 60 en ingreso per cápita (146.2 miles de pesos).

La Zona Metropolitana de Tehuacán (ZMTH), por su parte, es la segunda en importancia al contar con 296 mil 899 habitantes ubicados en una superficie de 647.3 kilómetros cuadrados y presentar una densidad media urbana de 73.2 habitantes por hectárea. Esta zona se encuentra conformada por los municipios de Tehuacán (274 mil 906 habitantes) y Santiago Miahuatlán (21 mil 993 habitantes) que se ubican al sureste de la entidad. En tanto, la Zona Metropolitana de Teziutlán (ZMTZ) presenta una población de 122 mil 500 habitantes en una superficie de 240.9 kilómetros cuadrados y una densidad media urbana de 50.3 habitantes por hectárea. Los dos municipios que constituyen esta zona son Teziutlán (92 mil 246 habitantes) y Chignautla (30 mil 254 habitantes) que se ubican en la zona norte del estado. Los niveles de complejidad económica en estas dos ciudades son muy inferiores a los que se 
observan en el Estado en su conjunto y la Zona Metropolitana Puebla-Tlaxcala, por lo que sus indicadores de bienestar económico son un reflejo de las pobres capacidades productivas del interior de la entidad.

En síntesis, las principales causas del crecimiento urbano en el Estado de Puebla están relacionadas directamente con el desarrollo de la actividad industrial manufacturera, el comercio y los servicios, pero también con las altas tasas de natalidad y el incremento de la esperanza de vida derivados de progresos sustantivos en higiene, servicios de salud, campañas de prevención e introducción de agua potable. Asimismo, con la disminución del índice de mortalidad infantil, la creación de centros asistenciales de protección a la infancia, las campañas de vacunación, el uso de antibióticos y la mejora en los hábitos de alimentación. Las migraciones del interior del Estado han contribuido también al crecimiento urbano, por el desplazamiento de la población rural hacia zonas periféricas metropolitanas que son atraídas por mayores oportunidades de trabajo, acceso a la educación y la salud. La migración nacional, por su parte, es otro factor importante en el que se destacan grupos provenientes de diferentes comunidades del sureste y centro del país atraídos por la actividad económica y los mejores índices de seguridad pública. En el caso de los extranjeros, se han establecido diferentes comunidades en la zona metropolitana de PueblaTlaxcala al ser un importante centro de consumo que garantiza también altos niveles de vida.

De manera especial, el Estado de Puebla ha generado paulatinamente una vocación exportadora, posicionando a la entidad en el lugar No. 11 en la participación de exportaciones no petroleras a nivel nacional. Sin embargo, la mayoría se concentran en sectores y productos específicos, donde el $67.7 \%$ de las exportaciones totales (12 mil 156 millones de dólares) provienen del ensamblaje de automóviles y vehículos de transporte. En ese sector se destacan la rama de automóviles (53.1\%), autopartes y tractores (10.9\%) y vehículos para transporte de mercancías (3.65\%). El tercio de esas exportaciones tiene una 
composición mucho más variada, en las que se destacan el sector textil, los muebles rústicos y la maquinaria de diferente tipo. Adicionalmente, la creciente e intensa urbanización y metropolización han generado una mayor capacidad y competitividad en la prestación de servicios educativos, de salud, prestación de servicios turísticos, financieros y culturales.

\section{Sustentación teórica}

Para Blanco (2006), la inclusión social, la equidad de género y la igualdad de oportunidades son tres de los grandes desafíos de las democracias contemporáneas. La inclusión es un asunto de gran significación por su complejidad, multidimensionalidad y carácter público, que demanda intervenciones institucionales efectivas para garantizar su aplicación y asegurar el empoderamiento social. Ramos (2017:1) ha definido que la inclusión y la equidad de género son una agenda pendiente para los gobiernos "al ser ellos los responsables de que efectivamente todas las personas y grupos sociales asentados en un territorio determinado sean beneficiarios de los derechos jurídicamente establecidos y puedan ser circunscritos a procesos de sostenibilidad integralmente definidos". Del mismo modo, son también los encargados de construir mayores espacios de participaciòn y deliberaciòn pública en los que los ciudadanos puedan contribuir al desarrollo de una mejor vida democrática.

Mediante los procesos de gobernanza, dicen Benavente y Valdés (2014:32-33), "pueden generarse políticas públicas incluyentes en las que los ciudadanos puedan participar, sobre todo aquellos que se encuentran en explicitas condiciones de segregación o marginación, alcanzar mejores oportunidades económicas, sociales, políticas y culturales y satisfacer, en mayor medida, sus necesidades y aspiraciones". En esa condición, las autoras exponen la necesidad de generar políticas públicas participativas e incluyentes que se constituyan en fórmulas institucionales pertinentes para enfrentar con probidad la pobreza, la marginación, la desnutrición, el hambre, el desempleo, los rezagos educativos, la segregación étnica y las carencias de salud. De igual forma, "se constituyen en mecanismos propicios para enfrentar 
la intolerancia, la segregación y la censura, prevenir la corrupción y desterrar la impunidad, así como otras problemáticas socialmente extendidas".

En el Estado de Puebla, corresponde a los poderes federales, estatales y municipales establecer los marcos normativos y favorecer los planes y políticas de coordinación y financiamiento que compensen socialmente las diferencias y contradicciones generadas por un crecimiento económico desequilibrado y propicien la incorporación de todos los grupos sociales al espacio de oportunidades para que puedan beneficiarse de un mayor bienestar individual y colectivo (Aguilar, 2015). Por ello, resulta fundamental definir la importancia de la legitimidad política como principio básico de la gobernabilidad democrática y, sobre todo, la pertinencia de acordar con los diferentes grupos y comunidades los mejores modelos de gobernanza para enfrentar los rezagos sociales y culturales en los distintos territorios y sentar las bases para un desarrollo más armónico y sustentable en sus diferentes formas y modalidades. La voluntad de la ciudadanía expresada en las urnas ha sido explicita en las últimas elecciones y contundente en cuanto a las prioridades que debe contener la nueva agenda pública, así como las condiciones a que ha de someterse en los próximos años el quehacer gubernamental. En ese contexto, la sociedad reclama hoy acciones eficientes de combate y prevención de la corrupción, la impunidad y la simulación, intervenciones estratégicas que reduzcan la inseguridad, la violencia y la expansión del crimen organizado, políticas públicas equitativas que favorezcan el empleo, reduzcan sustantivamente los índices de pobreza y que garanticen mejores niveles de desarrollo humano.

Desde la perspectiva de Olvera y Arellano (2015:598) un gobierno incluyente "debe considerar la amplitud de regiones existentes y sus diferentes vocaciones que reclaman atenciones integrales debido a su diversidad cultural y condiciones naturales. En ese sentido, desde las actividades agrícolas primarias hasta los servicios financieros globalizados, desde el turismo en sus variadas dimensiones hasta la producción artesanal, desde los servicios 
educativos interconectados hasta los servicios básicos de salud, desde el ensamblaje automotriz y la industria manufacturera hasta una economía informal extendida, se requieren políticas públicas participativas que permitan a la sociedad civil organizada (empresas privadas, organizaciones profesionales, colectivos y sindicatos) contribuir en su implementación y evaluación para asegurar avances efectivos, beneficios tangibles y verdaderos resultados que mejoren el bienestar colectivo". El bienestar no es sólo de carácter material, sino que considera también conocimientos intelectuales y profesionales, competencias para el trabajo y la colaboración colectiva, valores democráticos y de convivencia y prácticas que abunden en mejores espacios de interacción social y gobernabilidad

En esa consideración, desde la perspectiva de autores como Álvarez y Monsiváis (2015), Del Tronco (2012) y Rodríguez (2008), un gobierno incluyente en materia política debe asegurar la libertad de expresión y la manifestación pública de las ideas sin censura y represión, promover el debate y la deliberaciòn sustentada, reducir la intolerancia y el respeto hacia las diferencias ideológicas, sociales y culturales, dar preeminencia a las necesidades colectivas sobre los intereses individuales, respetar los derechos de las minorías y garantizar espacios de convivencia social a través del deporte, la cultura y el ocio creativo De manera fundamental, como lo señala Meyer (2013), "establecer un nuevo contrato social que recomponga de manera consistente el tejido social, asegure el desarrollo integral de los niños y jóvenes, garantice la equidad de género, permita la participación y desarrollo de las personas con capacidades diferentes y dignifique la presencia social de los adultos mayores mediante el aprovechamiento de su experiencia y el otorgamiento de diferentes servicios con calidad".

Con base en estas consideraciones, una nueva agenda pública debería construirse desde la participación ciudadana y en consonancia con el beneficio de amplios sectores de la 
población, sin condicionamientos partidarios o ideológicos, limitación de recursos 0 acotamiento en la toma de decisiones (Gobierno de México, 2018). De igual forma, habría que dar paso a auténticos espacios de deliberación y acuerdo, con sustentos jurídicos de largo plazo y programas definidos por la rendición de cuentas y la evaluación de resultados y beneficios. Esos procesos de gobernanza democrática incluyente habrán de definir a los gobiernos como entidades de fomento y coordinación de esfuerzos, cuya legitimidad se sustente en la participación ciudadana y la deliberación pública, la aceptación social y la confianza pública. Ellos deberán constituirse en organizaciones que se complementen, comprometan y responsabilicen de la atención y solución de los problemas sociales y, sobre todo, reconozcan el servicio público como una práctica profesional sustentada en principios éticos y convicciones políticas e ideológicas de equidad, inclusión y bienestar colectivo (Cohn, 2015).

\section{Modelo de intervención}

El modelo de gobernanza local ha sido reconocida como una metodología pertinente, al sustentarse en experiencias y buenas prácticas internacionales cuyo propósito es impulsar el desarrollo integral de los gobiernos locales mediante un proceso de inclusión y participaciòn articulada de diversos actores. En el caso de Puebla, buscará la concurrencia del gobierno del Estado, las representaciones y agencias federales, los gobiernos municipales, las empresas y agrupaciones del sector privado y las organizaciones sindicales, campesinas y civiles. En ese escenario, los cabildos municipales serán las entidades encargadas de coordinar las oportunidades en el territorio a partir de un diagnostico en colectivo y el fomento del crecimiento económico sustentable con un enfoque de inclusión social.

En efecto, los ayuntamientos de Puebla, como entidades básicas de una estructura gubernamental federalizada, desempeñan un papel fundamental en las tareas de articulación, mediación y generación de consensos entre los diferentes actores del territorio. 
Su gestión reviste una importancia sustancial para generar las condiciones pertinentes y realizar una gestión eficiente que vincule al mismo tiempo las capacidades institucionales, los recursos financieros, materiales y humanos y las políticas, programas y estrategias con los actores políticos y sociales, sus potencialidades y recursos, para promover procesos de crecimiento económico y desarrollo humano requeridos por la población. El modelo propuesto tiene un enfoque sistémico, aglutinador, abierto y democrático que busca alcanzar el pleno ejercicio de la gobernanza y producir mejoras sustantivas en las condiciones de vida de las diversas comunidades que habitan el territorio particular. Sus herramientas favorecen una dinámica integral y ordenada, donde participan todos los componentes del proceso y a quienes se les demanda una constante interacción y compromiso. En el modelo cada factor es importante para el cumplimiento de los diferentes resultados, por lo que debe sustentarse en una autoevaluación periódica que evidencie las fortalezas y áreas de mejora del sistema en su conjunto. Cuenta también con principios enfocados a la satisfacción de los ciudadanos, situándolos como la razón de ser de todo el proceso y centro estratégico de la gestión municipal.

El modelo cuenta con un instrumento que ha sido ya experimentado en diferentes municipios del país. Sin embargo, es conveniente reajustar constantemente los procesos, incorporar buenas prácticas y adaptar las categorías a las condiciones políticas, económicas, culturales, geográficas y demográficas de cada región específica donde se aplique. En esa perspectiva, la herramienta puede constituirse en una perspectiva integradora que recabe evidencias y permita a los ayuntamientos y sus comunidades avanzar hacia una mejora continua de los procesos, el desempeño eficaz y resultados con significación. Con base en ello, los principios que hacen de este modelo un mecanismo eficaz para construir y generar gobernanza en los municipios de Puebla son: 
- Integralidad de enfoques- Aborda en forma sistémica el conjunto de factores que conducen a la obtención de determinados resultados. Entiende a la institución municipal como un organismo inteligente, que no solo procesa recursos para convertirlos en productos y servicios para la ciudadanía, sino que establece relaciones con su entorno, las modifica, aprende de esa modificaciones, reconfigura su propia estructura y forma de actuar y vuelve a relacionarse con el entorno de modo innovador y creativo. El sistema reconoce que cada parte de la organización influye en las otras, de modo que un funcionamiento óptimo es aquel en el que todas las partes actúan en consonancia con el todo y están alineadas a un mismo propósito explícito y común.

- Modelo basado en resultados. - Determina como se gestionan los procesos y de qué manera impactan en los indicadores que miden la efectividad, la productividad, la eficacia y la eficiencia de la acción pública. El uso de indicadores considera transformar los datos en información significativa, monitorear esa información y utilizarla para una toma de decisiones que impulse la mejora de los desempeño en resultados y multiplique los efectos sociales en forma constante.

- Autoevaluación sistemática. - Mide continuamente el desempeño de una manera formal y estructurada, de tal manera que entrega una puntuación global de la capacidad de gestión y permite conocer las fortalezas y principales áreas de mejora de cada proceso.

A partir de las evaluaciones es posible desarrollar planes de acción que se constituyan en componentes sustantivos de los procesos de mejora continua. Al mismo tiempo, estructura una evaluación pormenorizada y detallada del conjunto de procesos fundamentales de la organización, pero con capacidad para vincular los distintos aspectos que guardan relación. 
- Ciclo de mejora continua. - Apoya el ordenamiento estratégico de todos los ámbitos de la organización municipal, mediante buenas prácticas internas y el incremento del valor público que entrega tanto a sus usuarios directos como las demás partes interesadas de la organización (autoridades, ciudadanos, organizaciones y comunidad).

- Principios y valores. - Tiene como propósito fundamental satisfacer las necesidades de los diferentes grupos ciudadanos, considerando siempre las demandas específicas, diversidades y condiciones. Coloca al ciudadano en el centro de la gestión de los servicios públicos, impacta la forma de funcionamiento y el comportamiento general de toda la organización. El principio básico es que la acción institucional debe orientarse siempre a la atención/satisfacción/servicio de los ciudadanos y no sólo en los productos requeridos por la dinámica política. Para ello, es fundamental saber lo que necesita y requiere el ciudadano para, de esa manera, estructurar los servicios y diseñar procedimientos encaminados a una atención efectiva.

- Realidad territorial. - Reconoce que la diversidad territorial presenta siempre múltiples maneras en que las organizaciones municipales funcionan y cumplen con sus objetivos. En tal forma, constituye una guía de procesos valorativos y comparativos, considera la definición particular de políticas y valores, su sentido de visión, interpretación sobre el territorio y su población, el cometido como gobierno y las definiciones principales. Con base en estos conceptos se organizan las prácticas concretas que constituyen la gobernanza.

El enfoque establece también que cada gobierno municipal se ajuste a su propia realidad y desarrolle su gestión a partir de ocho criterios sustantivos. Todos ellos requieren de indicadores y estándares para ponderar la marcha de la organización municipal e impulsar los cambios para una mejora constante de resultados. Ellos son: 
- Liderazgo

- Ciudadanía

- Personal

- Gestión estratégica

- Planificación regional

- Gestión de recursos

- Información y conocimiento

- Resultados

- Liderazgo. - Examina como el equipo directivo contribuye a precisar, comunicar y compartir las definiciones políticas y estrategias los principios ontológicos y los objetivos del gobierno en una coyuntura de tiempo en particular. Analiza también como el equipo directivo toma las decisiones y conduce al gobierno para un desempeño relevante en el desarrollo territorial y el cumplimiento de metas, mediante una gestión que potencialice las fortalezas, reduzca las debilidades y mejore los resultados.

- Ciudadanía. - El rol de la ciudadanía como elector, usuario, beneficiario, participante vigilante y colaborador responsable de las políticas públicas territoriales es fundamental. Por tanto, se examina la manera como el gobierno municipal se relaciona con la ciudadanía y las organizaciones públicas, privadas y sociales del entorno para conocer sus expectativas, demandas y necesidades, al tiempo que se evalúa la percepción que ellos tienen del desempeño del municipio y sus resultados. Este elemento pone atención en los procesos y mecanismos de participación, información, conocimiento y consideración de la opinion de la ciudadanía en la toma de decisiones. 
- Personal. - Analiza como el gobierno involucra, gestiona y desarrolla a su personal, sea directivo, de mandos medios, carrera, base y sindicalizado. Es sabido que este es un punto sensible en este tipo de gobiernos, porque con el tiempo se le han aumentado sus atribuciones jurídicas, diversificados sus responsabilidades administrativas y ampliado las asignaciones presupuestarias, sin que ello se haya traducido en la conformación y entrenamiento de equipos profesionalizados y técnicos de primera línea. Por ello, la importancia de analizar permanentemente de qué manera el gobierno municipal evalúa las habilidades y capacidades requeridas por su personal, como genera y apoya un ambiente de trabajo que conduce al alto desempeño, como previene los actos de impunidad, corrupción, discrecionalidad u omisión y, sobre todo, como contribuye al crecimiento de las personas y la organización para una mejora continua de los servicios al ciudadano.

- Gestión estratégica. - Al ser la gestión estratégica fundamental para la gobernanza municipal y factor detonante y promotor del desarrollo del territorio, examina la manera como la organización establece sus objetivos estratégicos, planes de acción y los proyectos o líneas de trabajo, incluyendo tanto la forma en que se definen las estrategias y planes como el proceso mediante el cual se implementan y da seguimiento a las políticas. Incluye la revisión y análisis de factores que concentran el principal papel del gobierno como director/coordinador de la agenda del desarrollo territorial mediante instrumentos de planificación y gestión que son de su exclusiva competencias (como el ordenamiento territorial, por ejemplo).

- Planificación regional. - Analiza cómo se realiza el proceso de planificación del desarrollo regional y formulan y actualizan las políticas, planes y programas. Asimismo, cómo se construyen los instrumentos indicativos del ordenamiento territorial, lleva a cabo la toma de decisiones sobre los proyectos de inversión y gestiona el proceso de inversión. 
En particular, indaga cómo se promueve el rol del sector privado en el territorio y realizan los programas de fomento el emprendimiento, la innovación y el crecimiento económico. De manera especial, como se realiza la articulación con los otros órdenes de gobierno para gestionar coordinadamente los diferentes programas de desarrollo sustentable con inclusión social.

- Gestión de recursos. - Analiza como el gobierno del municipio gestiona sus recursos internos. Contempla la manera como se realiza la administración, soporte y asesoría en tecnologías de información y comunicación, la gestión de los recursos financieros, los procesos de compras de bienes y servicios y la relación con los proveedores.

- Información y conocimiento. - Examina como el gobierno mide su desempeño organizacional y se gestiona la información necesaria para apoyar las operaciones diarias y la toma de decisiones. Considera la manera como se obtiene y recopila la información sobre los resultados de la institución en todos los ámbitos y, sobre todo, las diversas formas de difusión sean estas hacia el interior o el exterior, mediante las páginas de transparencia, las oficinas de prensa y vinculación con los organismos públicos, privados y sociales.

- Resultados. - Verifica los niveles de avance y logro en aquellas materias directamente vinculadas con la gestión municipal. Mide los principales indicadores que reflejan resultados globales en la satisfacción de la ciudadanía y el propio personal de la organización, el desempeño financiero, la calidad de los proveedores y avances en el impacto de las áreas de acción estratégica. Entre ellas se encuentra el desempeño operacional de los sistemas de trabajo, como la gestión de las inversiones, los programas de desarrollo social y cultural, el crecimiento económico y el fomento productivo. 
Estos criterios, que buscan diferenciar las capacidades institucionales, no se explican en sí mismos, sino que se organizan a partir de un concepto político-ideológico determinado, una interpretación social y económica de la realidad territorial, un diagnóstico de los desafíos, dilemas y oportunidades y una postura explicita para encarar las oportunidades de futuro. En ese escenario, la aplicación del modelo requiere analizar el desempeño tanto de los 8 criterios como de 26 indicadores y 70 prácticas de gestión. Cada uno de ellos requiere de una breve contextualización de las normativas que rigen al gobierno, por lo que cada nueva práctica implica un registro de los cambios y la razón de ellos.

\section{A manera de conclusión}

Las nuevas necesidades de la democracia participativa, incluyente y enfocada al bienestar hacen necesario aplicar modelos de gobernanza que, mediante la confluencia de diferentes actores, hagan posible comprometer proyectos de desarrollo local de auténtico beneficio social. En ese sentido, es importante la inducción y empoderamiento de los cabildos municipales y el personal directivo de los ayuntamientos, así como diversos ejercicios de capacitación del personal operativo responsable sobre los diferentes procedimientos y las cinco etapas específicas del modelo:

1. Realizar un diagnóstico integral del territorio para identificar las fortalezas y áreas de oportunidad en materia económica, productiva, social, ambiental y cultural y elabora un inventario de necesidades y demandas de los distintos grupos de población, las organizaciones privadas y sociales.

2. Consensar los resultados del diagnóstico entre el personal directivo del ayuntamiento, establecen los criterios generales, definen las prioridades y los indicadores de gestión. 
Ello implica un involucramiento directo del Presidente Municipal, todos los regidores, el síndico procurador y el personal involucrado directamente en la toma de decisiones.

3. Documentar los procedimientos y desempeño actual de cada una de las dependencias y establecen las prácticas que deben modificarse a partir de talleres con el personal directamente involucrado en cada proceso y la atención directa al ciudadano.

4. Ejecutar una primera evaluación integral del cumplimiento sistémico de los criterios, indicadores y prácticas de gestión. Este primer análisis permite un primer diagnòstico del funcionamiento del ayuntamiento y la estructuración de un plan de mejora que sirva de sustento para los próximos años.

5. Repetir la evaluación cada año durante la gestión municipal con el propósito de generar estudios comparativos sobre cada uno de los criterios, los indicadores y las prácticas en general.

\section{Referencias}

Aguilar, L. (2015).Gobernanza y política pública para la igualdad. Edición del Consejo Nacional para Prevenir la Desigualdad. Catedra UNESCO de Igualdad y No discriminación y la Universidad de Guadalajara. México. https://www.conapred.org.mx/documentos cedoc/Gobernanza\%20y\%20politica\%20public a\%20para\%20la\%20igualdad-Ax.pdf

Álvarez, Ch y Monsiváis, A. (2015, septiembre-diciembre). Democracia, capacidades deliberativas e inclusión política juvenil: el caso de Baja California, en Revista Mexicana de Ciencias Políticas y Sociales No. 225. Volumen LX. http://www.scielo.org.mx/scielo.php?script=sci arttext\&pid=S0185-19182015000300161

Benavente, M. C. y Valdés, A. (2014, octubre). Políticas públicas para la igualdad de género Un aporte a la autonomía de las mujeres. Edición de la Comisión Económica para América Latina y el Caribe (CEPAL) Santiago de Chile,. https://repositorio.cepal.org/bitstream/handle/11362/37226/1/S1420372 es.pdf 
Blanco, R. (2006). La equidad y la inclusión social: Uno de los desafíos de la educación y la escuela hoy, en Revista Iberoamericana sobre Calidad, Eficacia y Cambio en Educación No. 3. Volumen IV. Red Iberoamericana de Investigación Sobre Cambio y Eficacia Escolar. pp. 1-15. https://www.redalyc.org/pdf/551/55140302.pdf

Cohn, A. (2015, 29 de octubre). Un nuevo enfoque sobre las ciudades en que se incorpore el tema de la inclusión, en Banco Mundial News. Washington, D. C. https://www.bancomundial.org/es/news/feature/2015/10/29/a-new-approach-to-citiesincluding-inclusion

Consejo Nacional de Evaluación (2017). Puebla, indicadores de desigualdad. https://www.coneval.org.mx/coordinacion/entidades/Puebla/Paginas/desigualdad.aspx

Del Tronco, J. (2012, julio-diciembre). Las causas de la desconfianza política en México, en Perfiles Latinoamericanos Núm. 40. Facultad Latinoamericana de Ciencias Sociales. pp. 227-251. https://www.redalyc.org/pdf/115/11523037009.pdf

García A. (2017).Empresas, actores sociales e instituciones en la organización productiva del territorio y la innovación para el desarrollo local. Edición de la Universidad Nacional Autónoma de México y la Asociación Mexicana de Ciencias para el Desarrollo Regional.

Gobierno de México. Sociedad incluyente. https://www.gob.mx/conadis/articulos/sociedadincluyente

Harvard University \& Massachusetts Institute of Technology (2016). Atlas de Complejidad Económica. $\quad$ https://www.gob.mx/se/mexicocompetitivo/es/articulos/mexico-sube-unaposicion-en-el-indice-de-complejidad-economica-2016?idiom=es

Índice de Progreso Social. Resumen ejecutivo por estado. México (2017). www.socialprogressimperative.org

Instituto Nacional de Geografía e Informática. Encuesta intercensal (2015). https://www.inegi.org.mx/programas/intercensal/2015/

Meyer, L. (2013). Nuestra tragedia persistente: La democracia autoritaria en México. Editorial Debate/Random House Mondadori

Organización de las Naciones Unidas. Objetivos de Desarrollo del Milenio. Informe 2015. (2015). $\quad$ https://www.undp.org/content/undp/es/home/librarypage/mdg/the-millenniumdevelopment-goals-report-2015.html

Olvera, G. y Arellano, D. (2015, octubre-diciembre). El concepto de equidades y sus contradicciones: La política social mexicana, en Revista Mexicana de Sociología No. 4. Volumen LXXVII. Universidad Nacional Autónoma de México, pp. 581-609. https://www.redalyc.org/pdf/321/32141548003.pdf

Ornelas, J. (2012, enero-marzo). Volver al desarrollo, en Revista Problemas del Desarrollo No. 168. Volumen CXVIII, pp. 7-35. 
Programa de las Naciones Unidas para el Desarrollo (2017). Índices e indicadores de desarrollo

humano. http://hdr.undp.org/sites/default/files/2017 human development statistical update es.pdf

Ramos, G. (2017, 20 de junio). La equidad de género: una agenda pendiente para la inclusión, en Foro Forbes Mujeres Poderosas: El reto de trascender.. https://www.oecd.org/centrodemexico/medios/la-equidad-de-genero-una-agendapendiente-para-la-inclusion.htm

Rodríguez, L. (2008, mayo). Políticas de inclusión en México: Un análisis de marcos a la Iniciativa Todos a la Escuela, en Revista Mad No. 18. Departamento de Antropología. Universidad de Chile, pp. 39-56.

Secretaría de Gobernación (2010). Delimitación de Zonas Metropolitanas. Consejo Nacional de

Población. http://www.conapo.gob.mx/en/CONAPO/Delimitacion de Zonas Metropolitanas

Székely, M., C., Rodríguez, I. Flores, L. \& Mendoza, P. (2017). La pobreza en el Estado de Puebla: Evolución y perspectivas para una agenda de política social, en Documentos del Banco Mundial. Washington, D. C. http://documentos.bancomundial.org/curated/es/127581495604525447/text/M\%C3\%A9xic o-La-pobreza-en-el-estado-de-Puebla-evoluci\%C3\%B3n-y-perspectivas-para-unaagenda-de-pol\%C3\%ADtica-social.txt

\section{Forma de citar este artículo}

MEYER, J. A. y LUMBRERAS, M. (2018). "Modelos de gobernanza y desarrollo sustentable: Una propuesta instrumental para los municipios de Puebla", en Revista Pangea, Núm. 9, Pág. 22-42. Red Académica Iberoamericana de Comunicación. Recuperado el _ de de 2 de: http://www.revistapangea.org 